\title{
SEQUENTIAL EIGENFUNCTION EXPANSION FOR A PROBLEM IN COMBUSTION THEORY
}

\author{
M. AL-REFAI ${ }^{1}$ and K. K. TAM ${ }^{1}$
}

(Received 29 November, 2001; revised 5 March, 2002)

\begin{abstract}
A method of sequential eigenfunction expansion is developed for a semi-linear parabolic equation. It allows the time-dependent coefficients of the eigenfunctions to be determined sequentially and iterated to reach convergence. At any stage, only a single ordinary differential equation needs to be considered, in contrast to the Galerkin method which requires the consideration of a system of equations. The method is applied to a central problem in combustion theory to provide a definitive answer to the question of the influence of the initial data in determining whether the solution is sub-or super-critical, in the case of multiple steady-state solutions. It is expected this method will prove useful in dealing with similar problems.
\end{abstract}

\section{Introduction and formulation}

A central problem in combustion theory is the initial-boundary value problem

$$
\begin{aligned}
\partial u / \partial t & =\nabla^{2} u+\delta e^{\alpha u /(\alpha+u)} ; \quad \underline{x} \in D, t>0, \\
u(\underline{x}, 0) & =h(\underline{x}) ; \quad u=0 \text { for } \underline{x} \in \partial D .
\end{aligned}
$$

Here $u$ is the temperature, $\delta$ is a positive parameter whose magnitude incorporates the ambient temperature, heat of reaction and the size of the material, $\alpha$ is a positive parameter denoting the dimensionless activation energy of the material, typically $\alpha \geq 20 ; \underline{x}$ and $t$ are spatial and time variables. All quantities are considered nondimensionalised. The equation is considered in a bounded domain $D$. The derivation of the above equation can be found in Frank-Kamenetskiir[3], and discussions on the problem can be found in Gelfand [4], Parks [7], Sattinger [8], Boddington, Gray and Wake [1] and Tam [9], among others. It is known that for a given $D$ and a given

\footnotetext{
${ }^{1}$ Department of Mathematics and Statistics, McGill University, Burnside Hall, Room 1005, 805 Sherbrooke St. West, Monterals, Quebec H3A 2K5, Canada; e-mail: tam@math.mcgill.ca. (C) Australian Mathematical Society 2003, Serial-fee code 1446-1811/03
} 
set of boundary conditions, there are two demarcation values of $\delta, \delta_{e}$ and $\delta_{c r}$, such that when $\delta$ is less than $\delta_{e}$, the steady-state solution is $O(1)$ in magnitude. This state is called sub-critical. When $\delta$ is greater than $\delta_{c r}$, the steady-state solution is $O\left(e^{\alpha}\right)$ in magnitude. This state is called super-critical. When $\delta_{e}<\delta<\delta_{c r}$, there are two stable steady states, one sub-critical and one super-critical, and the initial value determines which one is reached. In 1980, Tam [9] posed the question: for $\delta$ in the range $\delta_{e}<\delta<\delta_{c r}$, how large must the initial value be for the solution to be super-critical? An attempt at the answer was made in Tam [9], using approximations on the integral equation obtained from the differential equation, and in Tam [5], using a two-step linearisation approximation on the differential equation. Recently, Tam et al. [11] introduced the idea of Sequential Eigenfunction Expansion to study an elliptic problem associated with (1.1). In principle, the iteration scheme produces the exact solution, subject only to computational restrictions. In this study, we extend the idea to parabolic equations, and use it to provide a definitive answer to the question posed in 1980. We observe that in contrast to the phenomenon of solution bifurcation due to parameter variation which is usually studied, the present situation is one of initial value induced bifurcation.

In the next section, we explain the Sequential Eigenfunction Expansion procedure and consider the contraction property of the iteration. In Section 3, we use the procedure to construct the solution to the combustion problem, thereby providing the answer to the posed question. We then conclude with some comments and observations.

\section{Sequential eigenfunction expansion}

2.1. Introduction In this section, we are concerned with the solution of the semilinear parabolic equation

$$
\frac{\partial u}{\partial t}=\nabla^{2} u+f(u), \quad t>0, \underline{x} \in D
$$

subject to the initial condition $u(\underline{x}, 0)=h(\underline{x})$ and homogeneous boundary conditions of Dirichlet or Robin type. In general, the solution has to be obtained numerically, using finite difference or finite elements. However, if the domain $D$ is simple enough such that the set of eigenfunctions associated with the Laplacian, the domain and the homogeneous boundary condition is known, then the spectral method can be used in which the solution is sought as an expansion of the eigenfunctions with time-dependent coefficients. The coefficients are then determined from an infinite system of first-order non-linear ordinary differential equations obtained by using some closure conditions, with initial conditions derived from $u(\underline{x}, 0)=h(\underline{x})$. An obvious advantage of the 
spectral method is that the solution is amenable to further analysis. To proceed numerically, the infinite system has to be truncated to a finite $N$-dimensional system, and if the closure condition is to require $P u=\partial u / \partial t-\nabla u-f(u)$ to have zero projection on the first $N$ coordinates of the space spanned by the eigenfunctions, then the method is attributed to Galerkin and sometimes referred to as a projection method. It is clear that in the Galerkin method, the approximation improves with increasing $N$, but the numerical solution of the $N$-dimensional system becomes increasingly difficult. Even with robust programs, such as Auto (see [2]), to carry out the integration, the nonlinearity can make the integration very difficult, if not impossible, for $N$ sufficiently large. For this reason, we ask if it is possible to develop an alternate method, in which the size of $N$ does not play such an important role. We recognise that the difficulty with large $N$ stems from Galerkin's requirement that $P u$ should have zero projection on the first $N$ coordinates of the eigenfunction space simultaneously. To alleviate the situation, we relinquish the requirement of simultaneity, and compensate for it with iteration. We calculate the coefficients of the eigenfunctions sequentially, using the condition of zero projection of $P u$ sequentially, and then through iteration achieve the condition of zero projection of $P u$ on the $N$ eigenfunction coordinates. Indeed, the sequential nature of the computation makes the size of $N$ somewhat immaterial, as only a single equation is solved at any stage of the procedure. We develop the expansion method in Section 2.2, proof of convergence in Section 2.3, and use it in Section 3 to study the influence of the initial data for the problem posed in Section 1.

2.2. Sequential eigenfunction expansion We present the methodology in a general setting for the prototype parabolic equation

$$
P u=\frac{\partial u}{\partial t}-\nabla^{2} u-f(u)=0 ; \quad \underline{x} \in D, t>0,
$$

subject to the initial condition

$$
u(\underline{x}, 0)=h(\underline{x})
$$

and homogeneous boundary condition $A u=0$ of the Dirichlet or Robin type. We suppose the set of eigenvalues $\left\{\lambda_{i}\right\}$ and the normalised eigenfunctions $\left\{\phi_{i}\right\}$ associated with the Laplace operator, the domain and the homogeneous boundary conditions have been obtained. The restriction to the above types of boundary conditions is to ensure that the eigenvalues are all positive. Since our concern is with the construction procedure, we further suppose the existence of a solution has been established. Of course if we are able to construct a solution, its existence is demonstrated. We develop the expansion procedure in what follows and consider the contraction properties of the iterative process, but will leave the main part of the convergence proof to an Appendix in order to make the presentation easier to follow. Let $u(\underline{x}, 0)=h(\underline{x})=\sum_{i=1}^{\infty} c_{i} \phi_{i}(\underline{x})$ and let $\bar{u}$ be a generic symbol for an approximation to $u$. 
We first take $\bar{u}=a_{1}^{(0)}(t) \phi_{1}(\underline{x})$. We have

$$
P \bar{u}=\phi_{1} \frac{d a_{1}^{(0)}}{d t}+\lambda_{1} a_{1}^{(0)} \phi_{1}-f\left(a_{1}^{(0)} \phi_{1}\right) .
$$

In general $P \bar{u} \neq 0$, but we ask that $P \bar{u}$ has zero projection on $\phi_{1}$. Denoting the inner product of two functions $f$ and $g$ by $(f, g)$, the condition $\left(P \bar{u}, \phi_{1}\right)=0$ yields the equation

$$
\frac{d a_{1}^{(0)}}{d t}+\lambda_{1} a_{1}^{(0)}-\left(f\left(a_{1}^{(0)} \phi_{1}\right), \phi_{1}\right)=0
$$

which, together with $a_{1}^{(0)}(0)=c_{1}$, determines $a_{1}^{(0)}(t)$. The quantity $P \bar{u}$ is referred to as the residual. Our condition is that the expansion of $P \bar{u}$ in terms of the eigenfunctions $\left\{\phi_{i}\right\}$ should have zero first component. We next take $\bar{u}=a_{1}^{(0)} \phi_{1}+a_{2}^{(0)} \phi_{2}$ and we ask that $\left(P \bar{u}, \phi_{2}\right)=0$ be satisfied to give

$$
\frac{d a_{2}^{(0)}}{d t}+\lambda_{2} a_{2}^{(0)}-\left(f\left(a_{1}^{(0)} \phi_{1}+a_{2}^{(0)} \phi_{2}\right), \phi_{2}\right)=0
$$

which together with $a_{2}^{(0)}=c_{2}$, determines $a_{2}^{(0)}(t)$. Proceeding in this manner, we obtain a sequence of functions $a_{i}^{(0)}(t), i=1, \ldots, \infty$, where $a_{i}^{(0)}(t)$ is determined by solving

$$
\begin{aligned}
& \frac{d a_{i}^{(0)}}{d t}+\lambda_{i} a_{i}^{(0)}=\left(f\left(a_{1}^{(0)} \phi_{1}+\cdots+a_{i}^{(0)} \phi_{i}\right), \phi_{i}\right), \\
& a_{i}^{(0)}(0)=c_{i} .
\end{aligned}
$$

We denote $u^{(0)}=\sum_{i=1}^{\infty} a_{i}^{(0)}(t) \phi_{i}(\underline{x})$ and consider $u^{(0)}$ as the initial approximation to the solution $u$. Writing $f\left(u^{(0)}\right)=\sum_{i=1}^{\infty} b_{i}^{(0)}(t) \phi_{i}(x)$, we have

$$
P u^{(0)}=\sum_{i=1}^{\infty}\left\{\frac{d a_{i}^{(0)}}{d t}+\lambda_{i} a_{i}^{(0)}-b_{i}^{(0)}\right\} \phi_{i}(x) \text {. }
$$

Let

$$
\delta_{i}^{(0)}=\frac{d a_{i}^{(0)}}{d t}+\lambda_{i} a_{i}^{(0)}-b_{i}^{(0)} .
$$

In general, $\delta_{i}^{(0)} \neq 0$ and so $P u^{(0)} \neq 0$. When $P u=0$ the exact solution for (2.1) and (2.2) is obtained. We seek to modify $a_{i}^{(0)}$, with the aim of making $\delta_{i}^{(0)}=0$. However, if $a_{i}^{(0)}$ is changed, $b_{i}^{(0)}$ will change accordingly. We therefore introduce an iterative scheme as follows. We construct a new sequence $\left\{a_{i}^{(1)}(t)\right\}$ from the solution of

$$
\begin{aligned}
& \frac{d a_{i}^{(1)}}{d t}+\lambda_{i} a_{i}^{(1)}-b_{i}^{(0)}=0, \\
& a_{i}^{(1)}(0)=c_{i}
\end{aligned}
$$


from which we obtain $u^{(1)}=\sum_{i=1}^{\infty} a_{i}^{(1)}(t) \phi_{i}(x)$ and $f\left(u^{(1)}\right)=\sum_{i=1}^{\infty} b_{i}^{(1)} \phi_{i}(x)$. Proceeding in this manner, we obtain the $n^{\text {th }}$ iterate for $u$

$$
u^{(n)}=\sum_{i=1}^{\infty} a_{i}^{(n)}(t) \phi_{i}(\underline{x})
$$

where $a_{i}^{(n)}(t)$ is the solution of

$$
\begin{aligned}
\frac{d a_{i}^{(n)}}{d t}+\lambda_{i} a_{i}^{(n)} & =b_{i}^{(n-1)}(t), \\
a_{i}^{(n)}(0) & =c_{i},
\end{aligned}
$$

and $b_{i}^{(n)}(t)=\left(f\left(u^{(n)}(t)\right), \phi_{i}\right)$. Now it is clear that if the solution of (2.4) and (2.5) converges, then $P u^{(\infty)}=0$ and hence the exact solution will be obtained. If we multiply (2.4) by $e^{\lambda_{i} t}$ and integrate from 0 to $t$, we will have

$$
a_{i}^{(n)}(t)=e^{-\lambda_{i} t}\left\{c_{i}+\int_{0}^{t} b_{i}^{(n-1)}(\tau) e^{\lambda_{i} \tau} d \tau\right\}, \quad n \geq 1 .
$$

2.3. Convergence of the iterations To demonstrate the convergence of the iteration in (2.6), it is enough to show that

$$
\left|a_{i}^{(n)}(t)-a_{i}^{(n-1)}(t)\right| \leq C_{i}\left|a_{i}^{(n-1)}(t)-a_{i}^{(n-2)}(t)\right| \quad \forall t \in J=(0, T],
$$

for some $0<C_{i}<1$, that is, that there is a contraction on the iteration.

THEOREM 1. For each $i$ and $n$, where $n \geq m$ for some $m \in N$, let

$$
\begin{aligned}
& I_{i}^{(n)}(\tau)=\int_{D}\left\{f\left(u^{(n-1)}\right)-f\left(u^{(n-2)}\right)\right\} \phi_{i} d \underline{x}, \\
& f_{1 i}^{(n)}(\underline{x}, \tau)=\max \left\{\left(u^{(n-1)}-u^{(n-2)}\right) \phi_{i}, 0\right\}, \\
& f_{2 i}^{(n)}(\underline{x}, \tau)=\min \left\{\left(u^{(n-1)}-u^{(n-2)}\right) \phi_{i}, 0\right\}, \\
& I_{1 i}^{(n)}(\tau)=\int_{D} f_{1 i}^{(n)} d \underline{x}, \quad I_{2 i}^{(n)}(\tau)=\int_{D} f_{2 i}^{(n)} d \underline{x},
\end{aligned}
$$

and $m_{i}^{(n)}(\tau)=I_{1 i}^{(n)} / I_{2 i}^{(n)} \cdot I f$

$$
\max _{\tau \in J}\left\{\left|\frac{m_{i}^{(n)}(\tau) \frac{\partial f}{\partial u}\left(\xi_{1 i}^{(n)}\right)+\frac{\partial f}{\partial u}\left(\xi_{2 i}^{(n)}\right)}{m_{i}^{(n)}(\tau)+1}\right|\right\}<\lambda_{1},
$$

then the sequence (2.6) is contractive. Here $\lambda_{1}$ is the first eigenvalue of the negative Laplacian and $\xi_{1 i}^{(n)}, \xi_{2 i}^{(n)} \in\left[\min \left(u^{(n-1)}, u^{(n-2)}\right), \max \left(u^{(n-1)}, u^{(n-2)}\right)\right]$, 


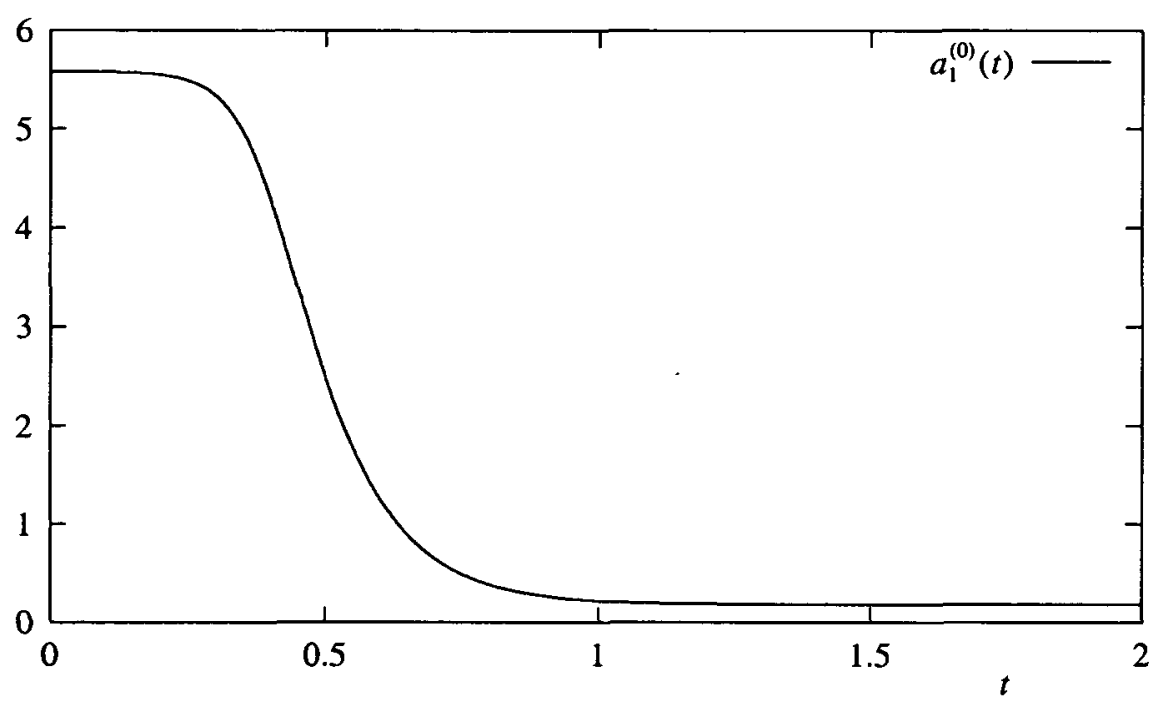

FIGURE 1. Here $a_{1}^{(0)}(t)$ for $A=5.581, \alpha=20$ and $\delta=1$.

The idea of the proof is to break up the integral into two parts so that the mean value theorem can be used in each part. We shall leave it to an Appendix so as not to impede the presentation of the main results. We emphasise that the convergence factor can only be assessed a posteriori. Further, since the condition of convergence is only a sufficient, and not a necessary, condition, numerical convergence serves as a more practical criterion.

\section{The combustion problem}

We now return to the problem posed in Section 1 . We take $D$ as the unit sphere. Assuming the solution depends on the radius only, the orthonormal eigenfunctions are $\phi_{n}=(1 / r \sqrt{2 \pi}) \sin (n \pi r)$ and the corresponding eigenvalues are $\lambda_{n}=$ $n^{2} \pi^{2}$, where $n=1,2, \ldots$ To begin with, let $h(r)=(A / \sqrt{2 \pi} r) \sin (\pi r)$, (where $\phi_{1}=(1 / \sqrt{2 \pi} r) \sin (\pi r)$ is the first normalised eigenfunction). If we write $h(r)=$ $\sum_{i=1}^{\infty} c_{i} \phi_{i}$, then $c_{1}=A$ and $c_{j}=0$ for $j \neq 1$. We fix $\alpha=20, \delta=1$. It is known from previous studies [5] that for these parameter values, (1.1) has two steadystate solutions. We use the expansion procedure to construct solutions for different values of $A$, and to determine the 'critical' value of $A$ separating the sub-critical and super-critical solutions. We also use the Galerkin method to study this problem and compare the numerical results. Numerical calculations for $a_{1}^{(0)}(t)$ indicate that for $A<5.581$, a steady-state solution is obtained and $a_{1}^{(0)}(\infty)$ approaches $0.18126015 \ldots$ 


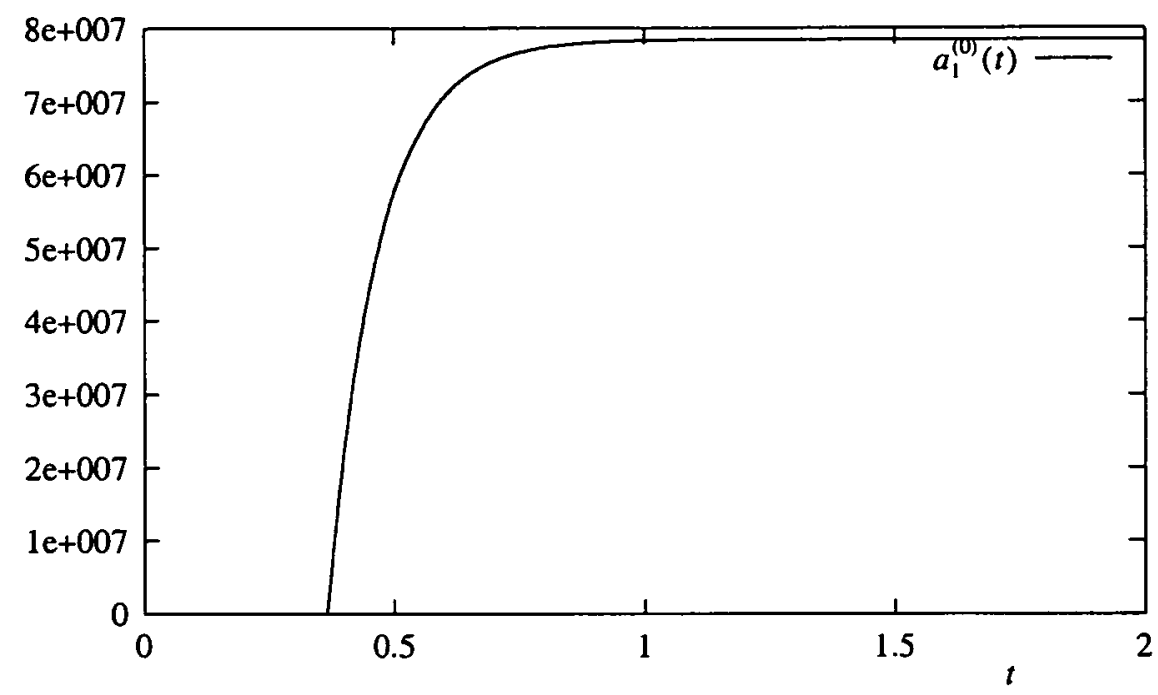

FIGURE 2. Here $a_{1}^{(0)}(t)$ for $A=5.582, \alpha=20$ and $\delta=1$.

which is of $O(1)$, and for $A>5.582$ another steady-state solution is obtained, and $a_{1}^{(0)}(\infty)$ approaches $78442987.109 \ldots$ So the critical point of $A$ will be between $5.581<A<5.582$. It is clear that the steady-state solution is not sensitive to perturbations in $A$, except when $A$ is near to its critical value. Figures 1 and 2 indicate the steady-state solutions of $a_{1}^{(0)}(t)$ at $A=5.581$ and $A=5.582$.

Using the Galerkin method, it is difficult to recognise the multiple solutions, since for each value of $A$ we have to solve a system of nonlinear ordinary differential equations.

We now consider the case of an arbitrary initial condition. In what follows, $u(x, 0)=h(x)$ is expanded in a series of eigenfunctions. If we write $h(x)=$ $\sum_{i=1}^{\infty} a_{i} \phi_{i}(\underline{x})$ then $a_{1}=\int_{D} h(x) \phi_{1}(x) d \underline{x}$. We know from the previous discussion that in the absence of all other coefficients, the sub-critical solution will be obtained if $a_{1}<5.581$, and if $a_{1}>5.582$, the super-critical solution will be obtained. Therefore the critical value depends on the first coefficient of the initial condition $u(\underline{x}, 0)$ in the eigenfunction expansion, when all other coefficients are small by comparison. As a specific example, we consider

$$
u(x, 0)=h(x)= \begin{cases}H & 0 \leq x \leq \epsilon \\ 0 & \text { otherwise }\end{cases}
$$

where $H$ and $\epsilon$ are parameters. We ask: what combination of $H$ and $\epsilon$ will determine if the solution is sub- or super-critical? 


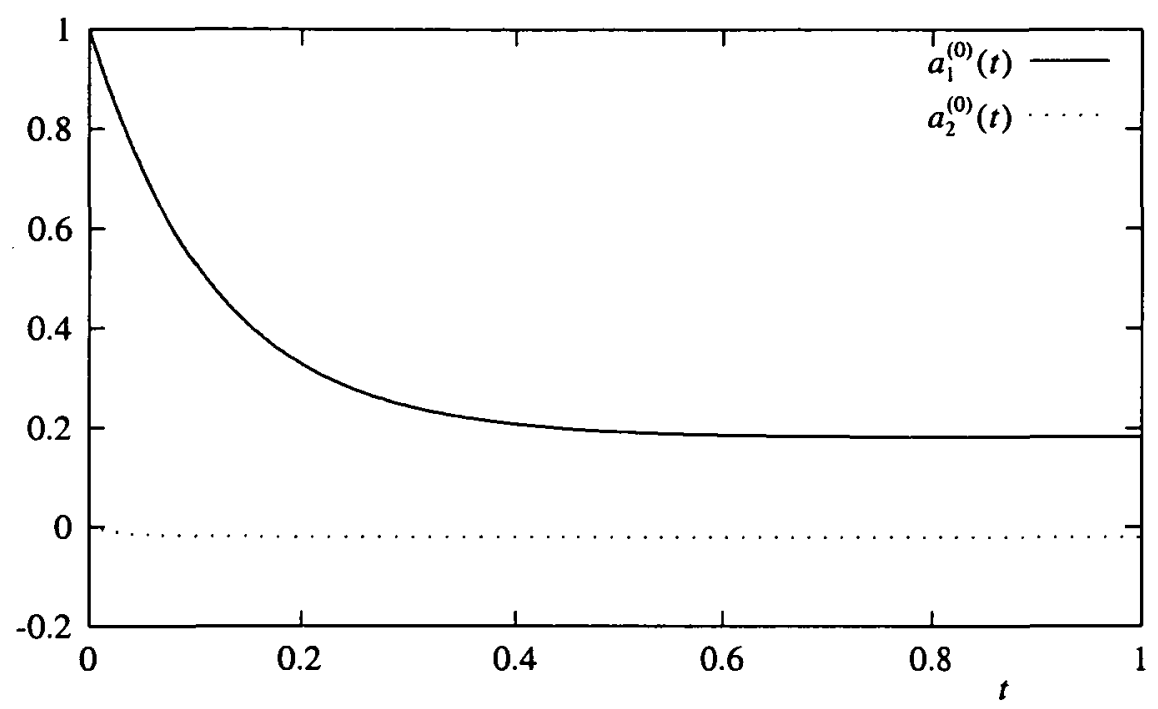

FIGURE 3. Comparison of $a_{1}^{(0)}(t)$ and $a_{2}^{(0)}(t)$ for $\delta=1, \alpha=10$ and $u(r, 0)=\sin (\pi r) /(r \sqrt{2 \pi})$.

Now

$$
\begin{aligned}
\int_{D} h(x) \phi_{1}(x) d \underline{x} & =\int_{\theta=0}^{2 \pi} \int_{\phi=0}^{\pi} \int_{x=0}^{1} h(x) \frac{1}{\sqrt{2 \pi}} \frac{\sin \pi x}{x} x^{2} \sin \phi d x d \phi d \theta \\
& =\frac{4 \pi}{\sqrt{2 \pi}} H \int_{0}^{\epsilon} x \sin \pi x d x \\
& =\frac{4 \pi H}{\sqrt{2 \pi}}\left\{\frac{\sin \pi \epsilon-\pi \epsilon \cos \pi \epsilon}{\pi^{2}}\right\} .
\end{aligned}
$$

Thus if

$$
\frac{4 H}{\pi \sqrt{2 \pi}}\{\sin \pi \epsilon-\pi \epsilon \cos \pi \epsilon\}>5.582,
$$

the solution is super-critical, and if

$$
\frac{4 H}{\pi \sqrt{2 \pi}}\{\sin \pi \epsilon-\pi \epsilon \cos \pi \epsilon\}<5.581,
$$

the solution is sub-critical. For $\epsilon \ll 1$, we have $\sin \pi \epsilon-\pi \epsilon \cos \pi \epsilon \sim \pi^{3} \epsilon^{3} / 3$. Thus ignition by a hot spot of radius $\epsilon$ requires an $H>1.063 \epsilon^{-3}$.

To verify that $a_{1}^{(0)}(t)$ is indeed the most important term in the sequence $\left\{a_{i}^{(0)}(t), i=\right.$ $1,2 \ldots\}$ and that the effect of the other terms on the solution $u$ is negligible we show in Figure 3 that the magnitude of $a_{2}^{(0)}(t)$ is very small compared with $a_{1}^{(0)}(t)$. The remaining coefficients are too small to be shown. 
TABLE 1. Comparison of $a_{i}(t)$ and $s_{i}(t), i=1, \ldots, 5$.

\begin{tabular}{|c|c|c|c|c|}
\hline \hline Method & $t$ & 0.25 & 0.50 & 1.00 \\
\hline Galerkin & $s_{1}(t)$ & 0.276728 & 0.191945 & 0.181294 \\
\hline \multirow{2}{*}{ Present } & $a_{1}^{(0)}(t)$ & 0.276964 & 0.192080 & 0.181397 \\
\cline { 2 - 5 } & $a_{1}^{(S)}(t)$ & 0.276759 & 0.191951 & 0.181294 \\
\hline \hline Galerkin & $s_{2}(t)$ & -0.020506 & -0.020672 & -0.020686 \\
\hline \multirow{2}{*}{ Present } & $a_{2}^{(0)}(t)$ & -0.020518 & -0.020679 & -0.020692 \\
\cline { 2 - 5 } & $a_{2}^{(s)}(t)$ & -0.020505 & -0.020672 & -0.020686 \\
\hline \hline Galerkin & $s_{3}(t)$ & 0.006040 & 0.006048 & 0.006049 \\
\hline \multirow{2}{*}{ Present } & $a_{3}^{(0)}(t)$ & 0.006043 & 0.006050 & 0.006050 \\
\cline { 2 - 5 } & $a_{3}^{(S)}(t)$ & 0.006040 & 0.006048 & 0.006049 \\
\hline \hline Galerkin & $s_{4}(t)$ & -0.002539 & -0.002540 & -0.002541 \\
\hline \multirow{2}{*}{ Present } & $a_{4}^{(0)}(t)$ & -0.002539 & -0.002541 & -0.002541 \\
\cline { 2 - 5 } & $a_{4}^{(5)}(t)$ & -0.002539 & -0.002540 & -0.002541 \\
\hline \hline Galerkin & $s_{5}(t)$ & 0.001298 & 0.001298 & 0.001298 \\
\hline \multirow{2}{*}{ Present } & $a_{5}^{(0)}(t)$ & 0.001298 & 0.001298 & 0.001298 \\
\cline { 2 - 5 } & $a_{5}^{(5)}(t)$ & 0.001298 & 0.001298 & 0.001298 \\
\hline \hline Galerkin & $s_{6}(t)$ & -0.000750 & -0.000751 & -0.000751 \\
\hline \multirow{2}{*}{ Present } & $a_{6}^{(0)}(t)$ & -0.000750 & -0.000751 & -0.000751 \\
\cline { 2 - 5 } & $a_{6}^{(j)}(t)$ & -0.000750 & -0.000751 & -0.000751 \\
\hline \hline
\end{tabular}

Finally, Table 1 makes a comparison between the solution obtained by the Galerkin method and the solution obtained by the current method, for different values of $t$. For $\delta=1, \alpha=10, u(r, 0)=(1 / r \sqrt{2 \pi}) \sin \pi r$ and $u(r, t)=\sum_{i=1}^{s} s_{i} \phi_{i}$. It can be seen that the initial solutions $a_{i}^{(0)}(t)$ are very close to the exact ones $s_{i}(t) i=1, \ldots, 6$. After 5 iterations of the current method, convergence of the iterations is achieved and the modified solutions $a_{i}^{(5)}(t)$ almost coincide with the exact ones. In fact, convergence is achieved up to $10^{-5}$ for $a_{i}^{(5)}(t)$, except for $a_{1}^{(5)}(t)$.

\section{Concluding remarks}

We have developed a sequential eigenfunction expansion method for a semi-linear parabolic equation, and used it to assess the influence of the initial data in a combustion problem. With minor modifications, the method can be used to assess the influence of nonhomogeneus boundary conditions, as well as on other similar problems. We have also demonstrated analytically that, under certain assumptions that must be verified $a$ posteriori, the iterative process is contractive. While the analysis provides assurance 
about the soundness of the process, the computation can be carried forward irrespective of the analytical aspects, and whether numerical convergence can be achieved is left to the numerical results. From this standpoint, it is clear that the method can be used for a broader class of problems.

\section{Acknowledgements}

Al-Refai acknowledges the support of the Jordan University of Science and Technology, and McGill University, for the financial support received during the course of this study.

\section{Appendix A. Proof of Theorem 1}

From (2.6) we have

$$
a_{i}^{(n-1)}(t)=e^{-\lambda_{i} t}\left\{c_{i}+\int_{0}^{t} b_{i}^{(n-2)}(\tau) e^{\lambda_{i} \tau} d \tau\right\}, \quad n \geq 1
$$

Subtract (A.1) from (2.6) to get

$$
\begin{aligned}
a_{i}^{(n)}-a_{i}^{(n-1)} & =e^{-\lambda_{i} t} \int_{0}^{t} e^{\lambda_{i} \tau}\left(b_{i}^{(n-1)}(\tau)-b_{i}^{(n-2)}(\tau)\right) d \tau \\
& =e^{-\lambda_{i} t} \int_{0}^{t} e^{\lambda_{i} \tau} \int_{D}\left\{f\left(u^{(n-1)}\right)-f\left(u^{(n-2)}\right)\right\} \phi_{i} d \underline{x} d \tau \\
& =e^{-\lambda_{i} t} \int_{0}^{t} e^{\lambda_{i} \tau} I_{i}^{(n)}(\tau) d \tau .
\end{aligned}
$$

Using the mean value theorem, we have

$$
f\left(u^{(n-1)}\right)-f\left(u^{(n-2)}\right)=\frac{\partial f}{\partial u}\left(u^{(n-1)}-u^{(n-2)}\right),
$$

and hence

$$
\begin{aligned}
I_{i}^{(n)}(\tau) & =\int_{D} \frac{\partial f}{\partial u}\left(u^{(n-1)}-u^{(n-2)}\right) \phi_{i} d \underline{x} \\
& =\int_{D} \frac{\partial f}{\partial u} f_{1 i}^{(n)} d \underline{x}+\int_{D} \frac{\partial f}{\partial u} f_{2 i}^{(n)} d \underline{x},
\end{aligned}
$$

where $f_{1 i}^{(n)}(\tau) \geq 0$ and $f_{2 i}^{(n)}(\tau) \leq 0$. Applying the mean value theorem for integration to $(\mathrm{A} .3), I_{i}^{(n)}(\tau)$ can be written as

$$
I_{i}^{(n)}(\tau)=\frac{\partial f}{\partial u}\left(\xi_{1 i}^{(n)}\right) \int_{D} f_{1 i}^{(n)}(\tau) d \underline{x}+\frac{\partial f}{\partial u}\left(\xi_{2 i}^{(n)}\right) \int_{D} f_{2 i}^{(n)}(\tau) d \underline{x}
$$


where $\xi_{1 i}^{(n)}, \xi_{2 i}^{(n)} \in\left[\min \left(u^{(n-1)}, u^{(n-2)}\right), \max \left(u^{(n-1)}, u^{(n-2)}\right)\right]$. Now, using (2.3) and the orthonormality property of $\phi_{i}$, we get

$$
\int_{D}\left\{u^{(n-1)}-u^{(n-2)}\right\} \phi_{i} d \underline{x}=\int_{D} \sum_{j=1}^{\infty}\left(a_{j}^{(n-1)}-a_{j}^{(n-2)}\right) \phi_{j} \phi_{i} d \underline{x}=a_{i}^{(n-1)}-a_{i}^{(n-2)}
$$

Consider $f_{1 i}^{(n)}>0$ and $f_{2 i}^{(n)}<0$ (this is the general case for $f_{1 i}^{(n)}$ and $f_{2 i}^{(n)}$ and we will deal with the case when $f_{1 i}^{(n)}$ or $f_{2 i}^{(n)}$ equals 0 later on). From (A.4) we obtain

$$
I_{i}^{(n)}(\tau)=\frac{\partial f}{\partial u}\left(\xi_{1 i}^{(n)}\right) I_{1 i}^{(n)}(\tau)+\frac{\partial f}{\partial u}\left(\xi_{2 i}^{(n)}\right) I_{2 i}^{(n)}(\tau)
$$

Using (A.5), we have

$$
\begin{aligned}
I_{1 i}^{(n)}+I_{2 i}^{(n)} & =\int_{D} f_{1 i}^{(n)} d \underline{x}+\int_{D} f_{2 i}^{(n)} d \underline{x} \\
& =\int_{D}\left\{u^{(n-1)}-u^{(n-2)}\right\} \phi_{i} d \underline{x} \\
& =a_{i}^{(n-1)}-a_{i}^{(n-2)} .
\end{aligned}
$$

With $m_{i}^{(n)}(\tau)=I_{1 i}^{(n)}(\tau) / I_{2 i}^{(n)}(\tau)$, we have two cases for $m_{i}^{(n)}(\tau)$ :

(1) $m_{i}^{(n)}(\tau)=-1$; then $I_{1 i}^{(n)}(\tau)=-I_{2 i}^{(n)}(\tau)$ or $a_{i}^{(n-1)}=a_{i}^{(n-2)}$ and so convergence is achieved and the iteration stops.

(2) $m_{i}^{(n)}(\tau) \neq-1$; then from (A.6) we have

$$
\begin{aligned}
I_{i}^{(n)}(\tau) & =\frac{\partial f}{\partial u}\left(\xi_{1 i}^{(n)}\right) I_{1 i}^{(n)}+\frac{\partial f}{\partial u}\left(\xi_{2 i}^{(n)}\right) I_{2 i}^{(n)} \\
& =\frac{\partial f}{\partial u}\left(\xi_{1 i}^{(n)}\right)\left\{I_{1 i}^{(n)}+I_{2 i}^{(n)}\right\}+\left\{\frac{\partial f}{\partial u}\left(\xi_{2 i}^{(n)}\right)-\frac{\partial f}{\partial u}\left(\xi_{1 i}^{(n)}\right)\right\} I_{2 i}^{(n)}
\end{aligned}
$$

Multiplying by $m_{i}^{(n)}$, we have

$$
\begin{aligned}
m_{i}^{(n)}(\tau) I_{i}^{(n)}(\tau)= & m_{i}^{(n)}(\tau) \frac{\partial f}{\partial u}\left(\xi_{1 i}^{(n)}\right)\left\{I_{1 i}^{(n)}+I_{2 i}^{(n)}\right\} \\
& +\left\{\frac{\partial f}{\partial u}\left(\xi_{2 i}^{(n)}\right)-\frac{\partial f}{\partial u}\left(\xi_{1 i}^{(n)}\right)\right\} I_{1 i}^{(n)}
\end{aligned}
$$

Also, by rearrangement, we obtain

$$
I_{i}^{(n)}(\tau)=\left\{\frac{\partial f}{\partial u}\left(\xi_{1 i}^{(n)}\right)-\frac{\partial f}{\partial u}\left(\xi_{2 i}^{(n)}\right)\right\} I_{1 i}^{(n)}+\frac{\partial f}{\partial u}\left(\xi_{2 i}^{(n)}\right)\left(I_{1 i}^{(n)}+I_{2 i}^{(n)}\right) .
$$


Adding (A.8) and (A.9) we have

$$
\left(m_{i}^{(n)}(\tau)+1\right) I_{i}^{(n)}(\tau)=\left\{m_{i}^{(n)}(\tau) \frac{\partial f}{\partial u}\left(\xi_{1 i}^{(n)}\right)+\frac{\partial f}{\partial u}\left(\xi_{2 i}^{(n)}\right)\right\}\left(I_{1 i}^{(n)}+I_{2 i}^{(n)}\right),
$$

and so, using (A.7), we obtain

$$
I_{i}^{(n)}(\tau)=\frac{m_{i}^{(n)}(\tau) \frac{\partial f}{\partial u}\left(\xi_{1 i}^{(n)}\right)+\frac{\partial f}{\partial u}\left(\xi_{2 i}^{(n)}\right)}{m_{i}^{(n)}(\tau)+1}\left(a_{i}^{(n-1)}-a_{i}^{(n-2)}\right) .
$$

Substituting in (A.2) we get

$$
a_{i}^{(n)}-a_{i}^{(n-1)}=e^{-\lambda_{i} t} \int_{0}^{t} e^{\lambda_{i} \tau} \frac{m_{i}^{(n)}(\tau) \frac{\partial f}{\partial u}\left(\xi_{1 i}^{(n)}\right)+\frac{\partial f}{\partial u}\left(\xi_{2 i}^{(n)}\right)}{m_{i}^{(n)}(\tau)+1}\left(a_{i}^{(n-1)}-a_{i}^{(n-2)}\right) d \tau .
$$

Let $t$ be in the interval $J=(0, T]$. We then have

$$
\begin{aligned}
& \left|a_{i}^{(n)}-a_{i}^{(n-1)}\right| \\
& \quad \leq \max _{\tau \in J}\left\{\mid \frac{\left.m_{i}^{(n)}(\tau) \frac{\partial f}{\partial u}\left(\xi_{1 i}^{(n)}\right)+\frac{\partial f}{\partial u}\left(\xi_{2 i}^{(n)}\right) \mid\right\} \max _{\tau \in J}\left\{\left|a_{i}^{(n-1)}-a_{i}^{(n-2)}\right|\right\} e^{-\lambda_{i} t} \int_{0}^{t} e^{\lambda_{i} \tau} d \tau}{\quad=\max _{\tau \in J}\left\{\left|\frac{m_{i}^{(n)}(\tau) \frac{\partial f}{\partial u}\left(\xi_{1 i}^{(n)}\right)+\frac{\partial f}{\partial u}\left(\xi_{2 i}^{(n)}\right)}{m_{i}^{(n)}(\tau)+1}\right|\right\} \max _{\tau \in J}\left\{\left|a_{i}^{(n-1)}-a_{i}^{(n-2)}\right|\right\} e^{-\lambda_{i} t} \frac{1}{\lambda_{i}}\left\{e^{\lambda_{i} t}-1\right\}}\right. \\
& \quad \leq \frac{1}{\lambda_{1}} \max _{\tau \in J}\left\{\left|\frac{m_{i}^{(n)}(\tau) \frac{\partial f}{\partial u}\left(\xi_{1 i}^{(n)}\right)+\frac{\partial f}{\partial u}\left(\xi_{2 i}^{(n)}\right)}{m_{i}^{(n)}(\tau)+1}\right|\right\} \max _{\tau \in J}\left\{\left|a_{i}^{(n-1)}-a_{i}^{(n-2)}\right|\right\} .
\end{aligned}
$$

Now (A.10) is valid for every $t \in J$, and hence

$$
\begin{aligned}
\max _{r \in J}\left\{\left|a_{i}^{(n)}-a_{i}^{(n-1)}\right|\right\} \leq & \frac{1}{\lambda_{1}} \max _{\tau \in J}\left\{\left|\frac{m_{i}^{(n)}(\tau) \frac{\partial f}{\partial u}\left(\xi_{1 i}^{(n)}\right)+\frac{\partial f}{\partial u}\left(\xi_{2 i}^{(n)}\right)}{m_{i}^{(n)}(\tau)+1}\right|\right\} \\
& \times \max _{\tau \in J}\left\{\left|a_{i}^{(n-1)}-a_{i}^{(n-2)}\right|\right\} .
\end{aligned}
$$

Therefore if

$$
\max _{\tau \in J}\left\{\left|\frac{m_{i}^{(n)}(\tau) \frac{\partial f}{\partial u}\left(\xi_{1 i}^{(n)}\right)+\frac{\partial f}{\partial u}\left(\xi_{2 i}^{(n)}\right)}{m_{i}^{(n)}(\tau)+1}\right|\right\}<\lambda_{1}
$$

the contraction of the iteration is obtained. Note that in (A.10) we have used the fact that the eigenvalues $\lambda_{i}$ form a positive increasing sequence. 
In the previous proof, we considered $f_{1 i}^{(n)}>0$ and $f_{2 i}^{(n)}<0$. A simple case is obtained if $f_{1 i}^{(n)}$ or $f_{2 i}^{(n)}$ equals zero. Then $\left(u^{(n-1)}-u^{(n-2)}\right) \phi_{i}$ is of one sign. Applying the mean value theorem for integration to (A.3) we have

$$
\begin{aligned}
l_{i}^{(n)}(\tau) & =\frac{\partial f}{\partial u}\left(\xi_{i}^{(n)}\right) \int_{D}\left(u^{(n-1)}-u^{(n-2)}\right) \phi_{i} d \underline{x}=\frac{\partial f}{\partial u}\left(\xi_{i}^{(n)}\right)\left\{a_{i}^{(n-1)}-a_{i}^{(n-2)}\right\} \\
\xi_{i}^{(n)} & \in\left[\min \left(u^{(n-1)}, u^{(n-2)}\right), \max \left(u^{(n-1)}, u^{(n-2)}\right)\right] .
\end{aligned}
$$

Thus, from (A.2), we have

$$
a_{i}^{(n)}-a_{i}^{(n-1)}=e^{-\lambda_{i} t} \int_{D} \frac{\partial f}{\partial u}\left(\xi_{i}^{(n)}\right)\left\{a_{i}^{(n-1)}-a_{i}^{(n-2)}\right\} e^{\lambda_{i} \tau} d \tau
$$

Let $t$ be in the interval $J:(0, T]$. We then have

$$
\begin{aligned}
\left|a_{i}^{(n)}-a_{i}^{(n-1)}\right| & \leq \max _{\tau \in J}\left\{\left|\frac{\partial f}{\partial u}\left(\xi_{i}^{(n)}\right)\right|\right\} \max _{\tau \in J}\left\{\left|a_{i}^{(n-1)}-a_{i}^{(n-2)}\right|\right\} e^{-\lambda_{i} t} \int_{0}^{t} e^{\lambda_{i} \tau} d \tau \\
& =\max _{\tau \in J}\left\{\left|\frac{\partial f}{\partial u}\left(\xi_{i}^{(n)}\right)\right|\right\} \max _{\tau \in J}\left\{\left|a_{i}^{(n-1)}-a_{i}^{(n-2)}\right|\right\} e^{-\lambda_{i} t} \frac{1}{\lambda_{i}}\left\{e^{\lambda_{i} t}-1\right\} \\
& \leq \frac{1}{\lambda_{i}} \max _{\tau \in J}\left\{\left|\frac{\partial f}{\partial u}\left(\xi_{i}^{(n)}\right)\right|\right\} \max _{\tau \in J}\left\{\left|a_{i}^{(n-1)}-a_{i}^{(n-2)}\right|\right\} \\
& \leq \frac{1}{\lambda_{1}} \max _{\tau \in J}\left\{\left|\frac{\partial f}{\partial u}\left(\xi_{i}^{(n)}\right)\right|\right\} \max _{\tau \in J}\left\{\left|a_{i}^{(n-1)}-a_{i}^{(n-2)}\right|\right\}
\end{aligned}
$$

and hence

$$
\max _{i \in J}\left\{\left|a_{i}^{(n)}-a_{i}^{(n-1)}\right|\right\} \leq \frac{1}{\lambda_{1}} \max _{\tau \in J}\left\{\left|\frac{\partial f}{\partial u}\left(\xi_{i}^{(n)}\right)\right|\right\} \max _{\tau \in J}\left\{\left|a_{i}^{(n-1)}-a_{i}^{(n-2)}\right|\right\} .
$$

Therefore if

$$
\max _{\tau \in J}\left\{\left|\frac{\partial f}{\partial u}\left(\xi_{i}^{(n)}\right)\right|\right\}<\lambda_{1}
$$

the contraction is obtained.

We emphasise that the conditions (A.11) and (A.12) which give rise to the contraction as demonstrated are sufficient conditions for the iteration (2.6) to converge, but they are not necessary.

\section{References}

[1] Boddington, P. Gray and G. C. Wake, "Criteria for thermal explosions with and without reactant consumption", Proc. Roy. Soc. Lordon Ser. A 385 (1983) 289-311. 
[2] E. J. Doedel and J. P. Kernèves, "AUTO: Software for continuation and bifurcation problems in ordinary differential equations", Appl. Math. Report, California Institute of Technology.

[3] D. A. Frank-Kamenetskir, Diffusion and heat transfer in chemical kinetics (Plenum Press, New York, 1959).

[4] I. M. Gelfand, "Some problems in the theory of quasi-linear equations", Amer. Math. Soc. Trans. Ser. 229 (1963) 295-381.

[5] K. K.Tam, "Criticality dependence on data and parameters for a problem in combustion theory", J. Austral. Math. Soc. Series B 27 (1986) 416-441.

[6] K. K.Tam, "Critical parameters for some non-class $A$ configurations in combustion theory, with non-uniform boundary temperatures", J. Engin. Math. 21 (1987) 129-137.

[7] J.R. Parks, "Criticality criteria for various configurations of a self-heating chemical as functions of activation energy and temperature of assembly", J. Chem. Phys. 34 (1961) 46-50.

[8] D. H. Sattinger, "A nonlinear parabolic system in the theory of combustion", Quart. Appl. Math. 33 (1975) 47-61.

[9] K. K. Tam, "On the influence of the initial data in a combustion problem", J. Austral. Math. Soc. Series B 22 (1980) 193-209.

[10] K. K. Tam, "The temporal evolution of a system in combustion theory. II", Stud. Appl. Math. 79 (1988) 159-171.

[11] K. K. Tam, Andonowati and M. T. Kiang, "Nonlinear eigenfunction expansion for a problem in microwave heating", Canad. Appl. Math. Quart. 4 (1996) 311-325. 